\title{
Discussion on Teaching Reform of the Course "Real Variable Function and Functional Analysis"
}

\author{
Fan, Linyuan* \\ School of Statistics \\ Capital University of Economics and Business \\ Beijing, P. R. China \\ E-mail: fanlinyuan@cueb.edu.cn \\ *Correspending Author
}

\author{
Dou, Changsheng \\ School of Statistics \\ Capital University of Economics and Business \\ Beijing, P. R. China \\ E-mail: douchangsheng@cueb.edu.cn
}

\begin{abstract}
Through the practice teaching of the course Real "Variable Function and Functional Analysis", that most students find difficulties in learning, we discuss the cause, optimize the content, try different kinds of teaching methods, improve the examination system and correspondence with other mathematical courses.
\end{abstract}

Keywords-Real Variable Function; Functional Analysis; Teaching Methods; Teaching Reform; Pratical Methods.

\section{INTRODUCTION}

Real Variable Function and Functional Analysis is a required course for students of sophomore year and junior year who major in statistics or financial mathematics in college. In the autumn semester of 2014-2015, I have taught students who are major in financial mathematics and economic statistics this course. During my teaching, I noticed that most students are lack of passion in learning this course, for the simple reason that their mathematic analysis skill is not fully comprehended in the freshman year. Moreover, the core content of the course includes Lebesgue measure, integral theorem, space theory with algebra and geometric structure and operator theory that base on these spaces. There are a lot of abstract definition, theorem and demonstrations. The after class exercises are very difficult and technical, in other words, the methods of solving different questions are not the same at all, therefore, most students find it hard to learn it. Then, many students think that it's worthless studying this difficult and time consuming course, and some students even give up learning this course.

However, on the other hand, real variable function and functional analysis theory is one of the basic courses of modern higher mathematics, its importance is obvious. Students whose majors are financial mathematics and statistics have other courses like measure theory and stochastic theory and so on. For these students, no matter they choose to go to work after graduation or study for a master degree, it is an important basic course. So, how to make full use of class time to improve teaching standard and realize teaching goal has become a common problem to be solved.

\section{The Carefully Selected Teaching Content}

Currently there are myriads types of "Real Variable Function and Functional Analysis" textbook. Each university differentiates in terms of pedagogic goals and curriculum settings, thus, select a proper version is the priority of course arrangement and preparation.

In author's case, 48 class hours are given to impair the knowledge. The blind pursuit of the quantity, difficulty and comprehensiveness can easily lead to students' hard digestion and eventually, confusions on basic concepts. The students will have no ideas of what was learned in the past semester. Also, many basic concepts mentioned in class reflect themselves in sequent courses, thus, which content to be clearly impaired and which to be simply mentioned is decided by taking major construction and other elements into consideration. This course can be seen as the sequence and sublimation of math analysis in terms of content. Its original base is the measure theory. It takes up an important part in teaching and occupies $1 / 3$ of the volume of a traditional textbook, in which abstract definitions and theorems, complex construction processes, and massive quantity of inductions and deductions involve.

No matter how hard editors of the textbook work on the details, there is no radical change to the cumbersome content. Student will find them hard to understand, let alone apply them into real use. But is it necessary for a college student to fully understand the concept of Lebesgue measurable set right form the start? Is it true that one who acquired math analysis lessons well know of true number theory? Hu Shigeng said: if looking at the big picture, things are much easier than a complicated construction process. Measurement is no more than a natural generalization of the concept of length, area and volume. It can not only be used in handling regular figures that can be solved with old measurement, but sets of more complicated figures as well. And a measure of general measurable set can be by approximated by a simpler measure of set, just as using the area of a polygon to approximate a general flat area. In applying of measurement, measurable set and the concept of measure construction don't exert a lot of effect. The truly important 
thing is some properties of measure. Though the number is not big, they seem so natural. Once based on these basic properties, we can solve the problem effectively using measure.

What Mr.Hu said inspired us a lot. In real practice of teaching, we can directly deduce almost all of the theses out of the properties of Lebesgue measurement set, that is, not only time saving, but more receptive as well. With further explanation of examples, students can get intuitive understanding of abstract concepts. In the end, add the simplified proof and construction process so that the students can achieve a state of integrating.

\section{THE INTRODUCTION OF VARIOUS TEACHING METHODS}

\section{A. Introduction of New Problems}

When introducing new problems, one should pay attention to the connection between the things that are learnt and unlearnt. This will not only stimulate the passion of students in learning, but can also make it easier for them to get new knowledge.

For example, at the beginning of this lesson, students are curious about what the lesson is about, what the learning methods are and why they learn it. In the first lesson, one should explain directly, that the purpose of this class is to introduce a new integral. Then, according to the Riemann integral that was learnt in mathematical analysis class, a new question is put forward - why do we need to learn another integral? That is because Riemann integral is not good enough, in other word, the integrable range is not wide enough, which can not meet the demands in further studying in branch theories or using them. For instance, the value range of Dirichlet function in $[0,1]$ is 0,1 , which is quite simple in the view of value range, however, it is not integrable according to the definition of Riemann integral, therefore, a new integral should be introduced. What demands should the new integral meet? Apparently, the functions that follow the Riemann integrability are still integrable and their integral values should be equal, the functions that do not follow the Riemann integrability are integrable according to the new integral theory.

Secondly, the fundamental operation properties of the new integrals should still remain, such as linear operational, additivity, etc. By then, the Lebesgue integral will be introduced by the specific example of calculating the total value of paper currency. Riemann integral equals the sum of the value of every note, this calculating method is simple and fast when the number of notes is limited, however, when the number of the notes are infinite, the drawback of this method is apparent, therefore, the number of one-yuan note should be calculated first as $n_{1}$, then the two-yuan note will be calculated as $1_{2}, \ldots .$. . Finally, the sum of the banknotes is $\sum_{i-1}^{\prime \lambda} i \cdot n_{i}$. Using the methods of teaching like that not only help the students review the learnt knowledge, but also kindle their enthusiasm in learning.

\section{B. Using of Geometric Visual and PPT}

Use geometrical visual methods and teaching software in aiding teaching. For instance, in explaining the structure of Cantor set, we first introduce a closed interval $[0,1]$, then cut one third of it in the middle, which is $(1 / 3,2 / 3)-$ - two closed interval $[0,1 / 3]$ and $[2 / 3,1]$ are got, then we operate the same process repeatedly on these two minor closed intervals to infinity. This instruction seems difficult to understand, however, its essence is tricotomy. Using teaching software to illustrate the process will make it a lot easier for students to comprehend. Moreover, many conclusions in functional analysis are based on classical mathematical analysis. Therefore, we usually use the situations on plane to explain them, for example, using the circle in a plane to represent the sphere in Banach space and using the point range in space to represent the point range in Banach space. Although these may not be precise, they can illustrate the problems properly. What is more, they are much easier than the models in space, which makes teaching more effective. If these theorems and definitions are separated from geometrical visual, they will be very abstract and will not be easy to understand.

\section{Using of Seminar}

Introduce seminar into class. Seminar is a common teaching method among senior students and postgraduates. Its purpose is to give the leadership of the class to students. By professor asking questions, guiding and supporting, students become the leader of the class, analyzing the problem and solve the problem.

For example, after learning the theory of "the union of limited number of countable sets is still countable sets", one should let the students think whether the union of unlimited number of countable sets is still countable sets. The teacher should encourage the students to think actively, take part in the discussion and make wild guesses, even try to prove the hypotheses if it is possible and illustrate them to the class. After that, the teacher will explain the correct answer and the way to prove it. The more discussions are made, there will be more chances for teachers to change the inactive atmosphere into an active one and the students will be more interested in learning, therefore, students will have a further understanding of it.

\section{Carefully Select after Class Exercise}

Choose the after class exercises carefully, prepare for the exercise class. Another difficulty in teaching this lesson is that the after class exercise is hard, most of them are proof problems which needs abstract thinking. During the process of teaching, I found out that many students who learnt the mathematical analysis in freshman year are afraid of solving proof questions, and some of them give up in the exams and only do the calculations.

This may because on one hand, students are not adapt to the transformation of thinking from Elementary Mathematics to Higher Mathematics, on the other hand, students are not able to finish their homework easily for a long time, therefore, they are more likely to be under pressure and even be weary of studying. Thus, while choosing exercises, one should pay attention to the difficulty and the quantity of the questions instead of choosing difficult ones only. In the meantime, when having exercise classes, one should choose typical questions such as, exchanging order of limited theorem and integral, the using of theorems, etc., solving basic questions by using basic methods. Let the students solve them by themselves so that it can increase their sense of accomplishment. Besides, one should help the students practice their mathematical thinking, mathematic logical thinking and help them not only review what they already 
learnt, but also cultivate various abilities in mathematics as well.

\section{THE IMPROVEMENT OF ASSESSMENT METHODS OF COURSES}

As assessments are the examinations of teaching effect of a semester, the improvement of assessment methods is necessary. To have a better assessment of students' study, the author suggests that:

- Set up item pools. When choosing test questions, the difficulty and comprehensiveness should be cautiously considered.

- Lay stress on daily assessments. Things like participation in discussions in class, answer of questions raised by teachers, quality of homework completion and participation in group work to figure out some problems which is often assigned by the teacher can show how much have students gained.

- Combine different kinds of testing methods, such as written examinations, oral examinations, etc. For instance, oral examination is very popular abroad but not so often to be seen here domestic. In this kind of examination, students who attend the course set a proper time for exams with the teacher. At that day, he should enter the office facing the teacher alone. With one clerk present who will take notes on the whole process of this exam, the student will answer each question asked by the teacher. After that, teacher will give the final score shortly. This will directly show how the student himself understands what he learnt this semester.

\section{THE CONNECTION With Other SUBJECTS IN \\ MATHEMATICS AND DEVELOP STUDENT'S CREATIVITY}

In teaching Real Variable Function and Functional Analysis, instructing students to apply the theories studied in class to other relevant courses is also a kind of way to make students understand and use the theories better.

For instance, as modern probability theory is based on measure theory, to have a full understanding of axiom system in probability theory, the understanding of abstract measure in general space is necessary, which is also closely related to Lebesgue measure. Furthermore, after learning integration theory and measure theory, stochastic analysis can be introduced as well. Stochastic differential equation is an important tool in modern financial mathematics and statistics, which can be used in setting up models of financial derivatives such as futures, stocks and bonds and predicting some important economic situations and trends, and is accordant with the syllabus of the majors of financial mathematics and statistics.
Also, applying functional analysis to mathematic modeling, such as using variation principle to solve optimization problems, can not only let students know the theoretical significances of variation principle in application and increase students' interest in learning functional analysis, but also let students increase their courage and confidence in independent thinking and develop their creativities.

\section{CONCLUSION}

As an important compulsory major course, real variable function and functional analysis is an extension of mathematic analysis and a foundation of measure theory and stochastic analysis. The author hopes that the ideas discussed above, such as improving teaching methods and teaching effects, carefully selecting teaching content, using various teaching methods and improving assessment methods, can help the teacher realize a better teaching effect and help students develop mathematic thinking and creativity to provide a foothold for students to further study or job hunting.

\section{ACKNOWLEDGEMENT}

The author would like to thank all the editors.

\section{REFERENCES}

[1] Cheng, Qixiang and Zhang, Dianzhou, Basis of Real Variable Function and Functional Analysis, $3^{\text {rd }}$ Edition, China Higher Education Press, 2003.

[2] Zhou, Mingqiang, Real Variable Function, $2^{\text {nd }}$ Edition, Peking University Press, 2008.

[3] Hu, Shigeng, Real Variable Function, China Higher Education Press, 1999.

[4] Zhang, Xiaolan, Concise Tutorial of Real Variable Function and Functional Analysis, China Higher Education Press, 2004.

[5] Zheng, Weixing and Wang, Shengwang, Abstract of Real Variable Function and Functional Analysis, $3^{\text {rd }}$ Edition, China Higher Education Press, 2005.

[6] Deng, Donggao and Chang, Xinyi, Concise Tutorial of Real Variable Function, China Higher Education Press, 2005.

[7] Zhou, Xingwei, "Some expierence on Teaching Real Variable Function courses", Higher Science Education, Vol 1, 42-45, 2000.

[8] Bai, Meng, "On the Teaching of Real Variable Functions for the Financial Mathematics Majors", Journal of Zhaoqing University, Vol.33 No.2, 25-27, 2012.

[9] Song, Shuni, Zhang, Guowei and Wang, Xiaomin, Real Variable Function and Functional Analysis, China Higher Education Press, 2007.

[10] Tie, Yong, "College Students' Difficulty in Learning Real Variable Function and Teaching Reform", Journal of Qujing Normal University, Vol.30, No.3, 71-73, 2011.

[11] Xu, Xi'an, "Discussions on Teaching Methods of Functional Analysis", Studies in College Mathematics, Vol.11, No.1, 73-75, 2008.

[12] Cai, Liming, "Study on the teaching methods of real analysis", Journal of Science of Teacher's College and University, Vol.34, No.4, 66-69, 2014. 